\title{
ANGUILLICOLA CRASSUS, UN COLONISATEUR AGRESSIF RÉCEMMENT INTRODUIT DANS LES STOCKS EUROPÉENS D'ANGUILLES.
}

\author{
S.T. ASHWORTH (1), G. BLANC (2).
}

(1) Department of Biological Sciences, University of Exeter, Hatherly Laboratories, Prince of Wales Road, Exeter, EX4 4PS, United Kingdom.

(2) Laboratoire d'Aquaculture et Pathologie Aquacole, Ecole Nationale Vétérinaire de Nantes, Atlanpole - La Chantrerie, B.P. 40706, 44307 Nantes Cedex 03, France.

\section{RÉSUMÉ}

Dans les années 1980, le nématode parasite Anguillicola crassus, originaire d'ExtrêmeOrient, a été introduit en Europe, où il a conquis un nouvel hôte et de nouveaux milieux. Les données relatives à la distribution géographique d'Anguillicola crassus en Europe montrent une extension vaste et rapide de son aire de répartition. Bien qu'influencées par la géographie de la colonisation, les trajectoires actuelles des populations du parasite convergent vers une prévalence maximale, tandis que l'intensité parasitaire moyenne tend vers un plateau dans la plupart des biocénoses aquatiques continentales européennes. Ces données indiquent nettement que ce nématode possède de remarquables aptitudes à la dispersion. En milieu dulçaquicole, il possède également la capacité de se construire des populations abondantes quelques années après son introduction. Cette revue analyse les mécanismes biologiques qui ont permis cette invasion considérable: la survie du stade libre, la réceptivité des hôtes intermédiaires et paraténiques et la fécondité des adultes. L'épidémiologie descriptive de l'anguillicolose en Europe et l'impact potentiel sur le stock européen d'anguilles sont présentés dans le contexte des connaissances actuelles.

Mots-clés : Anguillicola crassus, Anguilla anguilla, anguillicolose, invasion biologique.

\section{ANGUILLICOLA CRASSUS, A RECENTLY INTRODUCED AGGRESSIVE COLONIZER OF EUROPEAN EEL STOCKS.}

\section{SUMMARY}

Conveyed from the Far East Asia, the nematode parasite Anguillicola crassus has been introduced into Europe and has successfully colonized most European countries throughout the 1980's. Although influenced by colonization patterns, parasite populations tend towards maximal prevalence and predictable mean intensities in most freshwater European biocenosis. These data indicate that this parasite possesses several attributes of an aggressive colonizer such as high dispersion ability, length of the free stage survival, high fecundity and reproductive rate, receptivity of intermediate and paratenic hosts. The biological mechanisms of the colonization of Anguillicola crassus and the parasite impact on the definitive host populations are reviewed.

Key-words : Anguillicola crassus, Anguilla anguilla, anguillicolosis, biological invasion. 


\section{INTRODUCTION}

Les transferts commerciaux d'espèces piscicoles d'eau douce ont conduit, comme on pouvait s'y attendre, à l'introduction parallèle d'un certain nombre de parasites qui leur étaient associés (KENNEDY, 1993). Anguillicola crassus, exemple de ce type de transfert, a colonisé une grande partie de l'Europe au cours de la décennie 1980 (BLANC, 1994; MORAVEC et TARASCHEWSKI, 1988 ; THOMAS, 1993).

Anguillicola crassus fut mis en évidence chez l'anguille européenne, Anguilla anguilla, en aquaculture au Japon dès 1969 (EGUSA, 1979). En effet, le déclin de la capture des civelles d'anguilles japonaises, Anguilla japonica, dans les années 1960, et l'augmentation de la demande aquacole ont conduit à un effondrement brutal de l'approvisionnement en civelles. Des civelles d'anguilles européennes capturées sur les côtes françaises furent alors importées par le Japon. Cependant, la productivité de l'anguille européenne fut mauvaise dans les structures d'élevage japonaises, en raison notamment d'une sensibilité élevée aux parasites suivants : Ichthyophthirius multifiliis, Pseudodactylogyrus sp. et Anguillicola crassus. Ce dernier est le seul de ces trois espèces qui soit natif et, probablement, endémique au Japon ; les deux autres espèces sont également originaires d'Extrême-Orient, mais sont cosmopolites.

Anguillicola crassus induit de sévères lésions de la vessie gazeuse chez l'anguille européenne et les individus infectés sont amaigris (EGUSA, 1979). Ce même auteur nous apporte la première certitude que l'infection de l'anguille européenne par Anguillicola crassus est associée à des conséquences pathologiques, alors que ce parasite ne semble pas induire d'effets pathogènes chez Anguilla japonica. De plus, il a attire l'attention sur les risques de l'introduction de ces parasites asiatiques en Europe: "Precautions should be taken to prevent the introduction of these parasites from Asia to Europe".

Néanmoins, Anguillicola crassus fut introduit en Europe lors d'importations d'anguilles japonaises originaires d'Extrême-Orient au début des années 1980 (KOIE, 1991; KOOPS et HARTMANN, 1989). A la suite de son introduction, Anguillicola crassus s'est disséminé rapidement à travers l'Europe en Allemagne (KOOPS et HARTMANN, 1989; PETERS et HARTMANN, 1986 ; TARASCHEWSKI, MORAVEC, LAMAH et ANDERS, 1987), Italie (CANESTRI-TROTTI, 1987), Pays-Bas (DEKKER et WILLIGEN VAN, 1989 ; KAMSTRA, 1990), Belgique (BELPAIRE, CHARLEROY (de), GRISEZ et OLLEVIER, 1990 ; BELPAIRE, CHARLEROY (de), THOMAS, DAMME VAN et OLLEVIER, 1989), Danemark (KOIE, 1988a; KOIE, 1988b), Hongrie (SZÉKELY, LANG et CSABA, 1991), Angleterre (KENNEDY et FITCH, 1990) et France (DUPONT et PETTER, 1988 ; FONTAINE, LE BELLE, LOPEZ, QUERAT, BARTHELEMY, SEBERT, ALINAT et PETTER, 1990) et une carte de cette expansion a été réalisée (BLANC, 1994).

II est probable que la détection de ce parasite fut en général simultanée de son introduction (KENNEDY, 1993), car cette détection est facilitée par la grande taille d'Anguillicola crassus, aisément identifiable lors d'un examen même rapide de l'hôte définitif. La vitesse et les modalités de l'extension de ce parasite à travers l'Europe résultent des transferts commerciaux sur l'ensemble du continent. Les transports d'anguilles vivantes conduisent à son introduction dans une nouvelle localité et ses attributs de colonisateur aboutissent à l'invasion rapide du nouveau milieu.

\section{SURVIE DU STADE LIBRE}

Une des caractéristiques biologiques d'Anguillicola crassus qui lui confère une bonne capacité de colonisation dépend certainement de la capacité de survie de la larve libre (L2) en milieu dulçaquicole, en fonction des facteurs variables de $\mathrm{pH}$, de salinité, de dureté calcique et de température (BLANC, 1994 ; KENNEDY, 1993 ; THOMAS et OLLEVIER, 1993b).

La quantité de réserves nutritives contenues dans les ovocytes d'Anguillicola crassus est supérieure à celle des ovocytes d'un autre Dracunculoidé, Philometra ovata. Anguillicola crassus est ovovivipare et la larve effectue sa première mue avant d'éclore. L'éclosion des oeufs 
d'Anguillicola crassus se produit entre 10 et $30^{\circ} \mathrm{C}$, le temps d'incubation étant inversement proportionnel à la température (THOMAS et OLLEVIER, 1993b). Les oeufs non éclos peuvent survivre l'hiver et éclore au printemps lors du réchauffement du milieu. La capacité des oeufs à survivre aux faibles températures pendant au moins 3 mois a des implications sur le transport d'anguilles vivantes infectées (THOMAS et OLLEVIER, 1993b). La salinité et la dureté calcique ont un effet négatif sur le taux d'éclosion, la survie larvaire et le pouvoir d'infestation, mais ne les suppriment pas, même dans l'eau de mer (BLANC, 1994 ; KENNEDY et FITCH, 1990).

L'éclosion libère une larve de deuxième stade, enveloppée par la cuticule de la $L 1$ qui est retenue. La larve L2 libre mesure entre 270 et $300 \mu \mathrm{m}$ de longueur (BLANC, BONNEAU, BAGIANTI et PETTER, 1992 ; MORAVEC, DI CAVE, ORECCHIA et PAGGI, 1993 ; PETTER, CASSONE et LE BELLE, 1990 ; PETTER, FONTAINE et LE BELLE, 1989).

\section{L'HÔTE INTERMÉDIAIRE EN EUROPE}

A l'éclosion, la larve L2 s'attache au substrat et ondule activement. Ses mouvements pendulaires et ondulatoires seraient à l'origine de l'attraction de l'hôte intermédiaire prédateur, mais ceci reste à prouver expérimentalement. Cette mobilité rendrait la larve attractive pour l'hôte intermédiaire, mais raccourcirait toutefois sa durée de vie (THOMAS et OLLEVIER, 1993b).

Le copépode cyclopidé Eucyclops serrulatus fut le premier hôte intermédiaire d'Anguillicola crassus signalé (EGUSA, 1979). Depuis, de nombreuses autres espèces de copépodes, mais également d'ostracodes, ont été infestées expérimentalement en laboratoire (PETTER et al., 1990) ; ceci indique leur participation potentielle au cycle d'Anguillicola crassus. La majorité des espèces d'hôtes intermédiaires étudiées avec succès sont des cyclopidés. Les ostracodes et les copépodes calanidés pourraient également être impliqués dans la transmission d'Anguillicola crassus.

Une fois ingérée par l'hôte intermédiaire, la larve pénètre dans son hémocoele à travers les parois du tube digestif, en perdant l'enveloppe cuticulaire de la L1. Dans I'hémocoele, la larve entreprend une deuxième mue en larve infestante du troisième stade (L3). La larve L3 peut atteindre une longueur de 876 à $972 \mu \mathrm{m}$ dans l'hôte intermédiaire (BLANC, 1994 ; MORAVEC et al., 1993 ; PETTER et al., 1989). La larve L3 peut être facilement distinguée de la larve L2 par la présence d'un appareil buccal sclérifié et d'un mucron terminal sur la queue (BLANC et al., 1992). La vitesse à laquelle la larve atteint le troisième stade est fortement influencée par la température (BONNEAU, BLANC et PETTER, 1991). A la température de $20-23^{\circ} \mathrm{C}$, la larve $\mathrm{L} 3$ se développe en 20 jours après l'infestation chez Cyclops strenuus et Acanthocyclops vernalis (MORAVEC et al., 1993).

Une fois le troisième stade atteint, la larve n'est pas seulement infestante pour l'anguille, mais également pour d'autres espèces de poissons lors de l'ingestion d'un hôte intermédiaire infesté.

\section{LES HÔTES PARATÉNIQUES}

HAENEN et BANNING VAN (1990) furent les premiers à mettre en évidence des larves d'Anguillicola crassus dans des poissons capturés dans un lac. Les larves du troisième stade furent observées chez l'éperlan (Osmerus eperlanus), le sandre (Stizostedion lucioperca), la grémille (Gymnocephalus cernuus), la perche (Perca fluviatilis) et l'épinoche (Gasterosteus aculeatus). Les gardons (Rutilus rutilus) et les brèmes (Abramis brama) examinés ne contenaient pas de larves d'Anguillicola crassus. SZÉKELY (1994) montra ensuite que le gardon et la brème pouvaient être considérés comme des hôtes paraténiques potentiels. CHARLEROY (de), CANNERTS, AUGUSTIJN, GRISEZ et BOON (1990) utilisèrent la carpe (Cyprinus carpio) comme hôte paraténique lors d'infections expérimentales de l'anguille, démontrant la capacité du stade infestant à utiliser une espèce différente de l'anguille comme hôte paraténique. THOMAS et 
OLLEVIER (1992) ont complété cette liste d'hôtes dulçaquicoles potentiellement paraténiques. Cependant, ils considèrent la perche comme un mauvais hôte paraténique en raison de la mue de la L3 dans cette espèce et de la perte d'infectivité qui en résulte. On peut donc se demander si la perche est un hôte paraténique ou un hôte définitif inadéquat. Une espèce marine, le gobie noir (Gobius niger), peut être considérée également comme hôte paraténique (HÖGLUND et THOMAS, 1992). Les efforts pour comprendre la dynamique de la transmission d'Anguillicola crassus en Europe doivent prendre en compte la capacité de ce parasite à utiliser des espèces d'hôtes paraténiques dulçaquicoles, estuariennes ou marines.

\section{L'HÔTE DÉFINITIF EN EUROPE}

Les cinq espèces d'Anguillicolidae ne parasitent que les membres du genre Anguilla. Anguillicola crassus a été signalé chez Anguillicola japonica (KUWAHARA, NIIMI et ITAGAKI, 1974) et Anguilla anguilla (EGUSA, 1979). Après l'ingestion d'un hôte intermédiaire infecté, la larve $L 3$ traverse les parois digestives pour rejoindre la vessie gazeuse, qui est en contact avec l'intestin et l'estomac (HAENEN, GRISEZ, CHARLEROY (de), BELPAIRE et OLLEVIER, 1989). 17 heures après l'infestation, les larves L3 sont trouvées dans les parois de la vessie gazeuse.

Une fois dans les parois de la vessie gazeuse, la larve mue au stade L4 puis mue en forme adulte lors de la pénétration dans la lumière de la vessie gazeuse. La larve L3 peut être distinguée de la $L 4$ par la présence de déirides, d'ailes latérales et par leur structure cuticulaire (BLANC et al., 1992). Cependant, ces caractères ne sont observables avec certitude qu'au microscope électronique à balayage (BLANC et al., 1992). Les adultes d'Anguillicola crassus restent libres dans la lumière de la vessie gazeuse et se nourrissent activement du sang de l'anguille. A la température du laboratoire, le temps minimal nécessaire pour le développement en stade adulte est d'un mois. Anguillicola crassus est sexuellement gonochorique et le temps minimal pour atteindre le stade gravide est compris entre 3 et 4 mois (THOMAS et OLLEVIER, 1993a). La femelle gravide d'Anguillicola crassus contient un grand nombre d'oeufs à un instant donné, un maximum de 150000 (THOMAS et OLLEVIER, 1993a). Ces mêmes auteurs considèrent qu'Anguillicola crassus se caractérise par une forte fécondité quand on la compare à d'autres Dracunculoïdea. Cependant, on ne sait pas encore comment la femelle d'Anguillicola crassus pond ses oeufs et la durée de la période patente n'est pas connue.

L'oeuf ou la larve L2 éclose passent ensuite de la vessie gazeuse au tractus digestif par le ductus pneumaticus et ainsi dans le milieu extérieur.

\section{CARACTÉRISTIQUES ÉPIDÉMIOLOGIQUES DE L'ANGUILLICOLOSE}

Deux points concernant les paramètres épidémiologiques de l'anguillicolose dans l'environnement dulçaquicole européen sont intéressants. Le premier est la prédictibilité apparente des niveaux de prévalence (pourcentage d'hôtes dans la population étudiée) et d'intensité moyenne (nombre moyen de parasites par hôte infesté). Le second est le niveau réel de l'intensité moyenne atteint et son évolution apparemment constante dans le temps. Même si l'on peut penser que l'on a détecté la présence d'Anguillicola crassus de manière contemporaine à son introduction, les prévalences parasitaires sont souvent déjà élevées et supérieures à $50 \%$. Cependant, alors que les prévalences augmentent rapidement jusqu'à un maximum de $100 \%$, le niveau d'intensité moyenne semble atteindre un plateau d'environ 4 nématodes adultes par anguille, qui persiste dans le temps (ASHWORTH, 1994). II est important de signaler cet effet sur les intensités moyennes, car c'est en fait le premier indice d'une régulation de la population du parasite.

La colonisation du Royaume-Uni illustre le mode de dispersion d'Anguillicola crassus dans le reste de l'Europe. L'exemple britannique montre comment le parasite forme des foyers d'infestation localisés à une aire géographique restreinte. Peu après sa détection en Angleterre, la répartition d'Anguillicola crassus était apparemment limitée à deux foyers d'infestation, ceux de la Tamise et de la Trent (KENNEDY et FITCH, 1990). Au début des années 1990, un autre 
foyer fut détecté dans le Sud-Ouest de l'Angleterre, dans l'Avon. Les transports commerciaux semblent logiquement à l'origine de ce nouveau foyer. Le foyer de la Tamise serait probablement dû à la libération fortuite de poissons infestés à partir du marché aux poissons de Billingsgate, celui de la Trent à la libération d'anguilles vivantes et d'oeufs d'Anguillicola crassus par les transporteurs d'anguilles, et celui de l'Avon par le transport d'anguilles aux alentours de Poole dans le Dorset. Quand le foyer est formé, les potentialités de colonisation d'Anguillicola crassus et les migrations naturelles de l'anguille étendent rapidement sa distribution. Les déplacements naturels de poissons infestés ainsi que les transferts de poissons entre rivières voisines par les pêcheurs d'anguilles ont probablement facilité cette dispersion secondaire. Le déplacement d'hôtes paraténiques infestés pour des raisons de repeuplement peut être également considéré comme un moyen ayant facilité la dispersion de ce parasite. Au Royaume-Uni, Anguillicola est à présent très largement répandu dans le NordEst, le Centre et le Sud-Ouest, et sa répartition n'est plus limitée aux foyers d'origine.

Anguillicola crassus a donc utilisé rapidement sa grande capacité de colonisation pour envahir les milieux dulçaquicoles européens; il a atteint des niveaux de prévalence élevés et des intensités moyennes prédictibles. Il est probable que des mécanismes auto-régulateurs maintiennent les populations de ce parasite à des niveaux d'intensités moyennes constants (ASHWORTH, 1994 ; BLANC, 1994). Les caractéristiques d'un colonisateur comme A. crassus peuvent le doter d'un potentiel pathogène (KENNEDY, 1994) et plusieurs auteurs ont cherché les effets pathogènes d' $A$. crassus sur l'anguille hôte.

\section{LA POSSIBILITÉ D'UNE MALADIE}

Comme indiqué précédemment, EGUSA (1979) a le premier signalé les effets pathogènes d'A. crassus chez l'anguille européenne. Les signalements concrets de mortalités d'anguilles associées à l'anguillicolose sont cependant limités aux systèmes d'élevages intensifs (HARTMANN, 1987; MELLERGAARD, 1988) et en milieu naturel au Lac Balaton caractérisé par une population d'anguilles de forte densité (MOLNAR, SZÉKELY et BASKA, 1991). Seules les infestations fortes semblent entraîner une maladie importante chez l'anguille d'élevage (KAMSTRA, 1990) ; cette maladie résulterait principalement d'infections bactériennes secondaires, et non de dommages directs causés par ce nématode. En milieu naturel, la mortalité d'anguilles dans le Lac Balaton serait due non pas directement à l'anguillicolose mais à l'association de l'infestation par Anguillicola et de températures estivales élevées dans un lac surpeuplé (MOLNAR et al., 1991). Les modifications lésionnelles induites par l'anguillicolose semblent principalement limitées à la vessie gazeuse et sont les suivantes : la muqueuse est hémorragique, les glandes gazeuses sont hypertrophiées et hyperhémiées; les parois de la vessie sont épaissies, ce qui entraîne un rétrécissement de sa cavité (MOLNAR, 1993). Ces modifications pathologiques sont notables chez les poissons fortement infestés mais restent à étudier de façon approfondie. Pour de faibles infestations, ces réponses lésionnelles ne sont pas observées. Cependant, SPRENGEL et LÜCHTENBERG (1991) ont montré que la vitesse de nage des anguilles infestées était fortement réduite ; cet effet est mesurable dès lors que l'hôte héberge un unique nématode. L'effet de l'anguillicolose sur les performances natatoires des anguilles pourrait avoir de sérieuses conséquences sur les populations d'anguilles européennes s'il affecte leur survie lors de leurs migrations vers la mer des Sargasses.

\section{CONCLUSION}

Anguillicola crassus est un bon exemple de parasite colonisateur qui a rencontré dans un environnement étranger un hôte très proche de son parent originel. L'anguille européenne, cependant, apparaît désavantagée car elle n'a jamais eu de contact avec ce parasite. Ce phénomène a été observé auparavant dans d'autres cas de rencontres poisson-parasite. Le parasite Nitzchia sturionis fut introduit dans la Mer d'Aral avec une espèce d'esturgeon importée, et a réussi à infecter l'espèce native, Acipenser nudiventris (BAUER, 1991). Le monogène Gyrodactylus salaris a causé des mortalités de parr de saumon atlantique, Salmo 
salar, en Norvège, après son introduction lors d'importations de salmonidés vivants (MO, 1994). A peine 15 ans ont été nécessaires à Anguillicola pour coloniser la plus grande partie du continent européen : l'accroissement de sa population est réellement une explosion démographique. La spécificité d'hôte n'a pas été une barrière à son expansion et il est probable que ce parasite atteindra l'Irlande et l'Amérique, où il rencontrera l'anguille américaine Anguilla rostrata. En Europe, l'impact du parasite sur les populations de l'hôte définitif reste incertain en raison du manque de données sur les effets pathogènes chez l'anguille, lors de sa migration de retour à travers l'Atlantique, en particulier. On peut affirmer, cependant, qu'Anguillicola crassus, une fois présent dans un milieu, infecte la majorité des populations d'anguilles et qu'il est connu pour ses effets pathogènes dans cette espèce.

\section{BIBLIOGRAPHIE}

ASHWORTH S.T., 1994. Possible regulation in the Anguillicola crassus host-parasite system. In Parasitic diseases of fish, Pike A.W. and Lewis J.W. Eds., Samara Publishing Limited, Tresaith, Dyfed, 141-150.

BAUER O.N., 1991. Spread of parasites and diseases of aquatic organisms by acclimatization: a short review. Journal of Fish Biology, 39 (5), 679-686.

BELPAIRE C., CHARLEROY (de) D., THOMAS K., DAMME VAN P., OLLEVIER F., 1989. Effects of eel restocking on the distribution of the swimbladder nematode Anguillicola crassus in Flanders, Belgium. J. Appl. Ichthyol, 5, 151-153.

BELPAIRE C., CHARLEROY (de) D., GRISEZ L., OLLEVIER F., 1990. Spreading mechanisms of the swimbladder parasite Anguillicola crassus in the European eel, and its distribution in Belgium and Europe. Int. Rev. gesam. Hydrobiol., 75 (6), 891.

BLANC G., 1994. Biologie du cycle d'Anguillicola crassus (Nematoda, Dracunculoidea), contrôle thérapeutique de ses populations. Thèse Doc. Univ. Académie de Montpellier, Université de Perpignan, Perpignan, 1-355.

BLANC G., BONNEAU S., BAGIANTI S., PETTER A.J., 1992. Description of the larval stages of Anguillicola crassus (Nematoda, Dracunculoidea) using light and scanning electron microscopy. Aquatic Living Resources, 5, 307-318.

BONNEAU S., BLANC G., PETTER A.J., 1991. Etude sur la biologie des premiers stades larvaires d'Anguillicola crassus (Nematoda, Dracunculoidea) : spécificité de l'hôte intermédiaire et influence de la température sur la durée du développement. Bull. Fr. Pêche Piscic., 320, 1-6.

CANESTRI-TROTTI G., 1987. Occurrence of the Nematode Anguillicola crassa Kuwahara, Niimi et Itagaki, 1974 in eels from the Po delta, Italy. Bull. Eur. Ass. Fish Pathol., 7 (5), 109-111.

CHARLEROY (de) D., CANNERTS V., AUGUSTIJN H., GRISEZ L., BOON J.H., 1990. An improved method for artificial infection of the European eel, Anguilla anguilla, with Anguillicola crassus (Nematoda, Dracunculoidea). J. Appl. Ichthyol., 6, 182-188.

DEKKER W., WILLIGEN VAN J., 1989. Short note on the distribution and abundance of Anguillicola in the Netherlands. Journal of Applied Ichthyology, 1, 46-47.

DUPONT F., PETTER A.J., 1988. Note ichtyologique : Anguillicola, une épizootie plurispécifique en Europe, apparition de Anguillicola crassa (Nematoda, Anguillicolidae) chez l'anguille européenne Anguilla anguilla en Camargue, Sud de la France. Bull. Fr. Pêche Piscic., $308,38-41$.

EGUSA S., 1979. Notes on the culture of the European eel (Anguilla anguilla, L.) in Japanese eelfarming ponds. Rapp. P.-V. Réun. Cons. int. Explor. Mer, 174, 51-58.

FONTAINE Y.A., LE BELLE N., LOPEZ E., QUERAT B.V., BARTHELEMY L., SEBERT P., ALINAT J., PETTER A.J., 1990. Infestation de populations françaises d'anguilles 
(Anguilla anguilla L.) par des nématodes (Anguillicola crassus) : essais thérapeutiques, évaluation des risques potentiels liés à l'écophysiologie de l'hôte. Ann. Parasitol. Hum. Comp., 65, 64-68.

HAENEN O.L.M., GRISEZ L., CHARLEROY (de) D., BELPAIRE C., OLLEVIER F., 1989. Experimentally induced infections of European eel Anguilla anguilla with Anguillicola crassus (Nematoda, Dracunculoidea) and subsequent migration of larvae. Dis. aquat. Org., 7, 97-101.

HAENEN O.L.M., BANNING VAN P., 1990. Detection of larvae of Anguillicola crassus in freshwater fish species. Aquaculture, 87, 103-109.

HARTMANN S., 1987. Schwimmblasenwürmer beim aal. Fischer und Teichwirt, 1, 2-3.

HÖGLUND J., THOMAS K., 1992. The black goby Gobius niger as a potential paratenic host for the parasitic nematode Anguillicola crassus in a thermal affluent of the Baltic. Diseases of Aquatic Organisms, 175-180.

KAMSTRA A., 1990. Anguillicola in Dutch eelfarms ; current state. Int. Rev. gesam. Hydrobiol., 75 (6), 867-874.

KENNEDY C.R., 1993. Introductions, spread and colonization of new localities by fish helminth and crustacean parasites in the British Isles : a perspective and appraisal. Journal of Fish Biology, 43, 287-301.

KENNEDY C.R., 1994. The ecology of introductions. In Parasitic diseases of fish, Pike A.W. and Lewis J.W. Eds., Samara Publishing Limited, Tresaith, Dyfed.

KENNEDY C.R., FITCH D.J., 1990. Colonization, larval survival and epidemiology of the nematode Anguillicola crassus, parasitic in the eel Anguilla anguilla, in Britain. Journal of Fish Biology, 36, 117-131.

KOIE M., 1988a. Parasites in eels, Anguilla anguilla (L.) from eutrophic Lake Esrum (Denmark). Acta Parasitologica Polonica, 33 (2), 89-100.

KOIE M., 1988b. Parasites in European eel Anguilla anguilla (L.) from Danish freshwater, brackish and marine localities. Ophelia, 29 (2), 93-118.

KOIE M., 1991. Swimbladder nematodes (Anguillicola spp.) and gill monogeneans (Pseudodactylogyrus spp.) parasitic on the European eel (Anguilla anguilla). J. Cons. int. Explor. Mer, 47, 391-398.

KOOPS H., HARTMANN F., 1989. Anguillicola infestations in Germany and in German eel imports. J. Appl. Ichthyol., 1, 41-45.

KUWAHARA A., NIIMI A., ITAGAKI H., 1974. Studies on a nematode parasitic in the air bladder of the eel. I. Description of Anguillicola crassa n. sp. (Philometridea, Anguillicolidae). Jpn. J. Parasitol., 23 (5), 275-279.

MELLERGAARD S., 1988. Alens svommeblaereorm Anguillicola en ny parasit i den europaiske alebestand. Nordisk Aquakultur, 4 (2), 50-54.

MO T.A., 1994. Status of Gyrodacty/us salaris problems and research in Norway. In Parasitic diseases of fish, Pike A.W. and Lewis J.W. Eds., Samara Publishing Limited, Tresaith, Dyfed.

MOLNAR K., 1993. Effect of decreased oxygen contents on eels (Anguilla anguilla) infected by Anguillicola crassus (Nematoda : Dracunculoidea). Acta Vet. Hung., 41 (3-4), 349-360.

MOLNAR K., SZÉKELY C., BASKA F., 1991. Mass mortality of eel in lake Balaton due to Anguillicola crassus infection. Bull. Eur. Assoc. Fish Pathol., 11 (6), 211-212.

MORAVEC F., TARASCHEWSKI H., 1988. Revision of the genus Anguillicola Yamaguti, 1935 (Nematoda : Anguillicolidae) of the swimbladder of eels, including descriptions of two 
new species, A. novaezlandiae sp. $n$. and A. papernai sp. n. Folia Parasitol., 35, 125-146.

MORAVEC F., DI CAVE D., ORECCHIA P., PAGGI L., 1993. Studies on the development of Anguillicola crassus Kuwahara, Niimi et Itagaki, 1974 (Nematoda: Dracunculoidea) in the intermediate host. Folia Parasitol., 40, 39-48.

PETERS G., HARTMANN F., 1986. Anguillicola, a parasitic nematode of the swimbladder spreading among eel populations in Europe. Dis. aquat. Org., 1 (3), 229-230.

PETTER A.J., FONTAINE Y.A., LE BELLE N., 1989. Etude du développement larvaire d'Anguillicola crassus (Dracunculoidea, Nematoda) chez un Cyclopidae de la région parisienne. Ann. parasit. Hum. Comp., 64, 347-355.

PETTER A.J., CASSONE J., LE BELLE N., 1990. Observations sur la biologie des premiers stades larvaires d'Anguillicola crassus, nématode parasite d'anguille. Ann. Parasit. Hum. Comp., 65, 28-31.

SPRENGEL G., LÜCHTENBERG H., 1991. Infection by endoparasites reduces maximum swimming speed of European smelt Osmerus eperlanus and European eel Anguilla anguilla. Dis. aquat. Org., 11, 31-35.

SZÉKELY C., 1994. Paratenic hosts for the parasitic nematode Anguillicola crassus in lake Balaton, Hungary. Dis. aquat. Org., 18, 11-20.

SZÉKELY C., LANG M., CSABA G., 1991. First occurrence of Anguillicola crassus in Hungary. Bull. Eur. Assoc. Fish Pathol., 11 (4), 162-163.

TARASCHEWSKI H., MORAVEC F., LAMAH T., ANDERS K., 1987. Distribution and morphology of two helminths recently introduced into European eel populations: Anguillicola crassus (Nematoda, Dracunculoidea) and Paratenuisentis ambiguus (Acanthocephala, Tenuisentidae). Dis. aquat. Org., 3, 167-176.

THOMAS K., 1993. The life cycle of eel parasite Anguillicola crassus (Nematoda : Dracunculoidea). Ph D thesis, Katholieke Universiteit, Leuven, Belgium, 1-157.

THOMAS K., OLLEVIER F., 1992. Paratenic hosts of the swimbladder nematode Anguillicola crassus. Dis. Aquat. Org., 13, 165-174.

THOMAS K., OLLEVIER F., 1993a. First estimate of the egg production of Anguillicola crassus (Nematoda: Dracunculoidea). Folia Parasitol., 40, 104.

THOMAS K., OLLEVIER F., 1993b. Hatching, survival, activity and penetration efficiency of second-stage larvae of Anguillicola crassus (Nematoda). Parasitology, 107, 211-217. 